Elsden, S. R. (1955). J. gen. Microbiol. 12, 332-336

\title{
Considerations of General Physiology
}

\author{
BY S. R. ELSDEN \\ Agricultural Research Council Unit for Microbiology, The University, Sheffield
}

Whilst morphological characters form the basis of the accepted systems of classification of bacteria, from the time of Orla-Jensen, attempts have been made to devise systems based primarily on the physiology or the biochemical modes of life of these organisms. This line of thought has had a permanent effect. An examination of the accepted generic names shows the influence of this approach; thus we have Propionibacterium, Desulphovibrio, Thiobacillus, Nitrosomonas, to instance but a few examples. At one time it was considered by many that certain types of physiology were not only useful aids to classification but had phylogenetic significance. In particular it was considered that autotrophic bacteria were primitive organisms and that increasing nutritional complexity indicated more highly evolved forms. The powerful arguments put forward by Haldane and by Oparin have made this hypothesis untenable and the question of the significance, if any, which can be attached to physiological properties has to be reconsidered.

The last few years have seen an immense improvement in the methods available for the separation and analysis of macromolecules of biological origin and in the methods of detection and estimation of cell constituents of low molecular weight. Application of these methods is still in its infancy, but sufficient work has been done already to suggest that the same structural pattern is found in all living things. Thus all cells so far examined appear to contain proteins, ribose nucleic acid and deoxyribose nucleic acid, all of which have the same basic structure and all of which are built up from the same components; the differences are differences in detail only. Likewise the same coenzymes seem to function in all cells. It is not surprising, therefore, that the synthesis of individual components of the cell proceed by mechanisms common to all cells in which the particular component is found. It is a fact that so far only very few synthetic pathways have been studied in detail, but wherever comparative studies have been made the answer is always the same. Thus, the mechanism of arginine synthesis is the same in animal tissues, Neurospora and Escherichia coli. Likewise the synthesis of serine appears to be similar in animal tissues and in bacteria and, more recently, studies of the synthesis of porphyrins have shown that the same set of reactions occurs in both animal tissues and in a variety of bacteria. Despite the enormity of the gaps in our knowledge I feel fairly confident that a given type of compound will be synthesized by the same mechanism in all organisms capable of achieving the synthesis of that compound. If this be so it is clear that, for the present at least, we cannot obtain any assistance in the building of a phylogenetic system of classification of bacteria from studies of synthetic mechanisms. 


\section{Considerations of general physiology}

The nitrogen requirements for growth are met either by elementary nitrogen or by nitrogen in some combined form. The ability to fix gaseous nitrogen is distributed in what appears to be an irrational way. Thus Azotobacter spp., various Clostridium spp., all the photosynthetic bacteria, the rhizobiumleguminous plant complex and certain blue-green algae fix nitrogen, and it is difficult to believe that these forms bear any close relationship to one another.

Carbon requirements are satisfied either by carbon dioxide, as in the case of the autotrophic bacteria or by carbon in organic combination in the case of the heterotrophs. Organisms are known which will grow upon oxalate and on formate, and in these cases it is impossible to decide at present whether the use of such oxidized forms of carbon is a manifestation of the autotrophic mode of life or whether the organisms concerned are heterotrophs. The ability to use carbon dioxide for growth is a property shared exclusively by the autotrophs and the green plants. On the other hand, the utilization of carbon dioxide for the synthesis of individual compounds, for example aspartic acid, is common to plants, bacteria and animals. This universal ability to fix carbon dioxide has tended to obscure the importance of the autotrophic bacteria, but the essential feature of the autotrophs is that, like the green plants, they satisfy all their requirements for carbon with carbon dioxide.

Recent work on the fixation of carbon dioxide in photosynthesis has shown that the mechanism is quite different from any previously described. We do not know how carbon dioxide is fixed in the chemosynthetic autotroph but, in the photosynthetic autotroph the mechanism seems to be the same as that found in the green plants; namely, one which involves the formation of 3-phosphoglyceric acid from a 2-carbon compound and carbon dioxide. Just how unique this mechanism is, is not known but, considering all the work which has been done of late on carbon dioxide fixation, it is perhaps significant that the formation of phosphoglyceric acid by carbon dioxide fixation has only been recorded in photosynthetic systems. Phosphoglyceric acid is such a stable substance and so easy to isolate that it would be difficult to overlook. It is important that the phosphoglyceric acid mechanism should be looked for not only in the chemosynthetic autotrophs but also in the heterotrophs and in animal tissues. Should it prove to be peculiar to the autotrophs and the green plants we would have information which might be of considerable phylogenetic significance.

Whilst the ability to metabolize a given compound is frequently used in the differentiation of two otherwise similar organisms no one would go so far as to claim that a close relationship existed between all organisms which metabolize a given compound. Such a conclusion would bring together all organisms which utilize benzoate and such a group would include certain strains of Azotobacter, Mycobacterium and Pseudomonas. Likewise, van Niel has indicated the difficulties which would ensue were all the organisms which utilize hydrogen sulphide grouped together; for this would mean the inclusion of such morphologically diverse organisms as Beggiatoa, Thiovulum, Thiobacillus and Chromatium; and, because of the similarity of the pigments, the Athiorhodaceae.

Basically two main energy sources are exploited for growth, viz. chemical 
energy and radiant energy. In the former instance chemical energy is ultimately made available by oxidation-reduction reactions and there are two types of process. First, there is the formation of high energy phosphate bond compounds at the substrate level. That is to say, at certain points along the metabolic chain phosphorylated compounds are produced which, in the presence of the appropriate enzyme, phosphorylate adenosine diphosphate giving adenosine triphosphate and the dephosphorylated intermediary. In the second method phosphorylation of adenosine diphosphate is coupled with the transfer of electrons from reduced pyridine nucleotides to oxygen via the cytochrome system. Whilst the mechanism of this process is not known, it is clear that the development of this system is of considerable evolutionary significance, increasing as it does the amount of energy which is available from a given substrate under aerobic conditions. Organisms which use oxygen as the first acceptor are termed aerobic and those which use other acceptors in the oxidation-reduction reactions are described as anaerobic. The distinction is a biochemical one but the terms are not always used in this sense. The terms anaerobe and anaerobic tend to imply a sensitivity to oxygen rather than the inability to use oxygen as a terminal acceptor. It would be preferable to define anaerobes as organisms with an anaerobic metabolism and aerobes as organisms with an aerobic metabolism; in this way the fundamental differences between the two groups of organisms are made clear. To differentiate by means of the trivial character of sensitivity to oxygen, which is difficult to define, is not helpful.

The phylogenetic significance of these two characters is doubtful. Organisms with an anaerobic metabolism are found in most families and it is probable that the ability to synthesize the cytochrome system can be lost without unduly jeopardizing an organism's chances of survival. Whilst there is evidence that the atmosphere of the earth was, for a considerable time, devoid of oxygen, there is no reason for believing that the present-day anaerobes are primitive organisms.

The utilization of radiant energy for growth is, in all cases, associated with the presence of a chlorophyll type of pigment; though chlorophyll is not in all cases the most active pigment. The various chlorophylls which have been described differ only slightly from one another in chemical structure and from what has been said above it is reasonable to believe that the mechanism of synthesis of these various chlorophylls will be essentially the same in all organisms. This raises the question of whether such a mechanism could have evolved independently in a number of unrelated organisms or whether it evolved once only. If the latter be accepted then, clearly, all organisms which contain chlorophyll must have descended from a common stock and chlorophyll can be taken as a useful indicator of phylogenetic relationship. On the other hand, if chlorophyll formation be an instance of parallel evolution then there is little more to be said.

In the animal kingdom creatine phosphate is found only in vertebrate muscle, in Balanoglossus, one of the Protochordata, and in the Echinoidea which are of course invertebrates. It is of interest that morphological and 
embryological evidence suggests that these organisms are related, and that the biochemical evidence supports this view. Perhaps chlorophyll is a biochemical indicator of phylogeny in much the same way as is creatine phosphate. Pringsheim has recently made a similar suggestion. The recently discovered diaminopimelic acid and related compounds may also be of use as indicators.

Haemoglobin is characteristic of vertebrate blood and occurs sporadically in the invertebrates. Until recently there was a tendency to believe that haemoglobin was peculiar to the animal kingdom. Recently, however, it has been found to occur in the nodules of leguminous plants and in yeast cells, and these observations might lead one to doubt the argument outlined above, namely, that possession of a particular compound indicates a common origin. However, I do not believe that this criticism is valid. Haemoglobin consists of a protein coupled with haem and there is a wide range of such compounds not all of which have the property of combining reversibly with oxygen; the enzymes catalase and peroxidase are good examples of this. The nature of the protein to which the haem is coupled seems to condition the properties of the resulting compound. It is thus not difficult to imagine haemoglobins cropping up in a wide variety of organisms purely by chance, for all that is required is a slight alteration in the protein synthesizing mechanism to obtain a protein which, when coupled with haem, produces a compound which can combine reversibly with oxygen. On the other hand, the synthesis of a molecule as complex as chlorophyll must involve the formation of a large number of enzymes whose actions must be co-ordinated, and the probability that such a system could have evolved more than once appears to me to be remote.

If it be accepted that all the chlorophyll-containing organisms have a common origin then it follows that the Athiorhodaceae, the Thiorhodaceae, the Chlorobacteriaceae and the green plants must have evolved from the same stock. Nothing is known of the detailed chemistry of the chlorophyll of the Chlorobacteriaceae; on the other hand, the chlorophylls found in the two groups of purple bacteria appear to be identical. In addition, both the Athiorhodaceae and the Thiorhodaceae have many morphological characters in common with the Pseudomonadaceae; and the Athiorhodaceae when grown aerobically in the dark cannot be easily distinguished from this group. The position of the Chlorobacteriaceae is not so clear-cut; their chlorophyll appears to differ from that of the other photosynthetic bacteria and their morphology is also quite distinct, for they are Gram-negative non-motile cocci. At the moment the most simple interpretation would appear to be that they are representatives of a line of evolution different from that of the Thiorhodaceae and the Athiorhodaceae.

The obvious morphological similarities between the two groups of purple bacteria on the one hand and the Pseudomonadaceae on the other suggest that they all belong to the same line of evolution. It may be a coincidence that most of the chemosynthetic autotrophic bacteria are characterized by being Gram-negative and with polar flagella when motile.

In view of this it is not too unreasonable to suggest that all the Gram- 
negative organisms with polar flagella have evolved from the same stock as the green plants in the following way. The photosynthetic bacteria have retained the ability to utilize radiant energy and carbon dioxide for growth. The autotrophic bacteria have retained only their ability to utilize carbon dioxide but obtain energy by oxidizing inorganic compounds; whilst the Pseudomonodaceae are heterotrophic, having lost the ability to utilize carbon dioxide and radiant energy. The Chlorobacteriaceae are taken to represent a second line of evolution from the common stock.

This argument hinges entirely on the assumption that chlorophyll was only evolved once. Studies on the mechanism of carbon dioxide fixation in autotrophic bacteria are urgently required, for should it prove that the mechanism is the same as that in photosynthetic organisms this would provide additional support to the above scheme. It is also important to investigate the distribution of the green plant carbon dioxide fixation mechanism, for should these reactions be more widespread than at present appears to be the case then the hypothesis would be weakened.

To conclude, I think it can be argued that in some instances physiological characters can be of use in the development of a phylogenetic system of classification, but so far the use is limited. The question of the relationship, if any, of the organisms already discussed, which do appear to fit into one unit, with the remainder of the Eubacteria, cannot be decided at present. We cannot decide because we have not sufficient evidence. This is a depressing admission, but it must not be concluded that a system of classification of bacteria which at the same time indicates phylogenetic relationships is impossible to attain. The only conclusion we can draw is that the present approach is inadequate. I have no doubt that, in time, characters will be discovered or rediscovered which, when their significance is appreciated, will enable us to build such a system. 